\title{
Tuberculous peritonitis in a German patient with primary biliary cirrhosis: a case report Yilin Vogel ${ }^{1}$, Jan C Bous ${ }^{1}$, Guido Winnekendonk ${ }^{2}$ and Bernhard F Henning*1
}

Address: ${ }^{1}$ Department of Internal Medicine, Gastroenterology Unit, Marienhospital, Ruhr University, Herne, Germany and ${ }^{2}$ Department of Radiology, Marienhospital, Ruhr University, Herne, Germany

Email: Yilin Vogel - yilin.vogel@marienhospital-herne.de; Jan C Bous - jan.bous@marienhospital-herne.de; Guido Winnekendonk - guido.winnekendonk@marienhospital-herne.de; Bernhard F Henning* - bernhard.henning@rub.de

* Corresponding author

Published: 31 January 2008

Journal of Medical Case Reports 2008, 2:32 doi:10.1 186/1752-1947-2-32
Received: 29 June 2007

Accepted: 31 January 2008

This article is available from: http://www.jmedicalcasereports.com/content/2/1/32

(C) 2008 Vogel et al; licensee BioMed Central Ltd.

This is an Open Access article distributed under the terms of the Creative Commons Attribution License (http://creativecommons.org/licenses/by/2.0), which permits unrestricted use, distribution, and reproduction in any medium, provided the original work is properly cited.

\begin{abstract}
Background: The number of cases of tuberculosis as a complication in people with immunodeficiency, people on immunosuppressive therapy and among the immigrant population is increasing in Germany. However, tuberculous peritonitis rarely occurs without these risks, particularly in Germans. The incidence of tuberculous peritonitis in Germany is very low; tuberculosis of the intestinal tract was found in approximately $0.8 \%$ of tuberculosis cases in 2004. The diagnosis of tuberculous peritonitis is often delayed on account of non-specific clinical symptoms. The absence of specific biological markers, long incubation times for cultures and nonspecific radiographic or ultrasonographic signs increase the morbidity associated with this treatable condition.
\end{abstract}

Case presentation: We report a case of tuberculous peritonitis in a 73-year-old female German patient. Her medical history revealed primary biliary cirrhosis (PBC) since 1992. On admission, she complained of abdominal pain, vomiting, ascites and peripheral edema. The patient has been in a seriously reduced general condition and had fever up to $39.6^{\circ} \mathrm{C}$. A few weeks earlier, the patient was in another hospital with the same complaint. Inflammatory parameters were elevated, but the procalcitonin level was normal. Blood culture was always negative, as was the tuberculin test. Ultrasonography of the abdomen showed massive ascites with multiple septa. The patient underwent a computed tomography (CT) scan of the abdomen which showed a thickened intestinal wall in the sigmoid colon and a pronounced enhancement of the peritoneum. Computed tomography scans of the lung showed only slight bilateral pleural effusion. Because of the anaesthetic and bleeding risk due to thrombocytopenia, laparoscopy was not immediately undertaken. The culture from ascites was positive for M.tuberculosis after three weeks.

Conclusion: In primary biliary cirrhosis patients with non-specific clinical symptoms, such as vomiting, abdominal pain, ascites, weight loss, and fever, tuberculous peritonitis must be considered in the initial differential diagnosis, although these symptoms may be attributed to cirrhosis of the liver with spontaneous bacterial peritonitis. Ultrasonographic and CT scab findings are not specific for tuberculous peritonitis, but an awareness of the ultrasonographic features and the features of the CT scan may help in the diagnosis of tuberculous peritonitis and avoid clinical mismanagement. 


\section{Background}

In industrialised countries, tuberculosis increasingly occurs in the immigrant population and in patients with acquired immune deficiency syndrome (AIDS) and those on immunosuppressive therapy. Tuberculosis of the intestinal tract ranked $8^{\text {th }}$ of all forms of tuberculosis $(0.8 \%)$ in 2004 in Germany, after pulmonary forms (79.6\%), extrathoracic lymph nodes $(7 \%)$, pleura $(3.6 \%)$, genitourinary $(3.3 \%)$, intrathoracic lymph nodes $(2.4 \%)$, osteoarticular $(1 \%)$, and spine $(0.9 \%)$. Tuberculous peritonitis is also rare in Germany. The diagnosis of any extrapulmonary forms of tuberculosis is quite difficult; in the case of peritoneal tuberculosis this is because clinical manifestations are non-specific, such as weight loss, abdominal pain, fever, ascites, vomiting [1-3]. The diagnosis of tuberculous peritonitis is often delayed on account of non-specific clinical signs or symptoms, absence of specific biological markers, long incubation times for cultures and non-specific radiographic or ultrasonographic signs. The prognosis in tuberculous peritonitis was unfavorable before treatment with antituberculous drugs became available and the mortality averaged 50 per cent [4].

\section{Case report}

Two months before the patient visited our hospital she had been admitted to the emergency unit of another hospital with vomiting, abdominal pain and weight loss of 10 $\mathrm{kg}$ within three months. A diagnosis of spontaneous bacterial peritonitis was ruled out. Her clinical signs were initially attributed to severe gastritis and an ulcer in the pyloric canal. She had suffered from primary biliary cirrhosis (PBC) since 1992 and had been treated with 750 mg of ursodeoxycholic acid daily without immunosuppressive therapy. She had no significant past history of pulmonary or genital tuberculosis. She had given birth to a son and a daughter.

Physical examination showed a blood pressure of 120/60 $\mathrm{mmHg}$; regular pulse at $84 / \mathrm{min}$; and a body temperature of $39.6^{\circ} \mathrm{C}$. Superficial lymph nodes were not palpable. Chest examination revealed basal breathing. The patient's abdomen was distended, and peristaltic sounds were not audible. Edema of the extremities was present. The initial laboratory data for blood (Table 1) rendered a high Creactive protein (CRP) level of $15.68 \mathrm{mg} / \mathrm{dl}$. Her tuberculin test was negative.

CT scan of the chest showed bilateral pleural effusions without lymph node swellings. Abdominal ultrasonography revealed massive ascites with multiple septa. A CT scan of the abdomen showed a thickened intestinal wall located in the sigmoid colon (Fig. 1) and pronounced enhancement of the peritoneum. There were no masses or lymph node swellings in the abdominal cavity. Esoph-
Table I: Laboratory data for blood on admission

\begin{tabular}{ll}
\hline WBC & $5.2 / \mathrm{nl}$ \\
Seg & $81 \%$ \\
Lymp & $8 \%$ \\
Mono & $8 \%$ \\
Eos & $0 \%$ \\
Baso & $2 \%$ \\
RBC & $3.64 / \mathrm{Pl}$ \\
Hb & $11.3 \mathrm{~g} / \mathrm{dl}$ \\
Ht & $34.2 \%$ \\
Platelets & $52 / \mathrm{nl}$ \\
Total protein & $8.4 \mathrm{~g} / \mathrm{dl}$ \\
Albumin & $1.7 \mathrm{~g} / \mathrm{dl}$ \\
GOT & $41 \mathrm{U} / \mathrm{l}$ \\
GPT & $16 \mathrm{U} / \mathrm{l}$ \\
LDH & $301 \mathrm{U} / \mathrm{l}$ \\
ALP & $217 \mathrm{U} / \mathrm{l}$ \\
Gamma-GT & $90 \mathrm{U} / \mathrm{l}$ \\
Total bilirubin & $1.7 \mathrm{ml} / \mathrm{dl}$ \\
CHE & $2057 \mathrm{U} / \mathrm{l}$ \\
CRP & $15.68 \mathrm{mg} / \mathrm{dl}$ \\
Creatinine & $0.67 \mathrm{mg} / \mathrm{dl}$ \\
Na & $135 \mathrm{mmol} / \mathrm{l}$ \\
K & $4.3 \mathrm{mmol} / \mathrm{l}$ \\
Lactate & $2.47 \mathrm{mmol} / /$ \\
AFP & $1.7 \mathrm{ng} / \mathrm{ml}$ \\
CA I25 & $67.4 \mathrm{U} / \mathrm{ml}$ \\
CA I9-9 & $33 \mathrm{Ulml}$ \\
CEA & $3 \mathrm{ng} / \mathrm{ml}$ \\
Quick & $45 \%$ \\
PTT & $45 \mathrm{Sec}$. \\
\hline
\end{tabular}

agogastroscopy and ileocoloscopy revealed no ulcer or stenosis in the colon or ileum.

The nature of ascites was revealed by puncture and findings are listed in Table 2, including a protein level of 4.6 $\mathrm{g} / \mathrm{dl}$. Microscopy was requested for malignant cells and Mycobacterium, neither of which was discovered. The cultures and polymerase chain reaction (PCR) analysis of stool and urine as well as from bronchial lavage were negative for M. tuberculosis, but the culture of ascites returned positive for M. tuberculosis after three weeks. The final diagnosis was tuberculous peritonitis.

We began anti-tuberculous therapy using isoniazid, rifampicin, ethambutol, and pyrazinamide.

In the end, the fulminating course of the disease could not be positively influenced by this therapy and multi-organ failure with liver failure and nephritic failure developed.

\section{Discussion}

Tuberculous peritonitis is always secondary to other tuberculous lesions. Tuberculous peritonitis appears to be more common in females than in males. Tuberculosis in females commonly reaches the peritoneum through tubal 


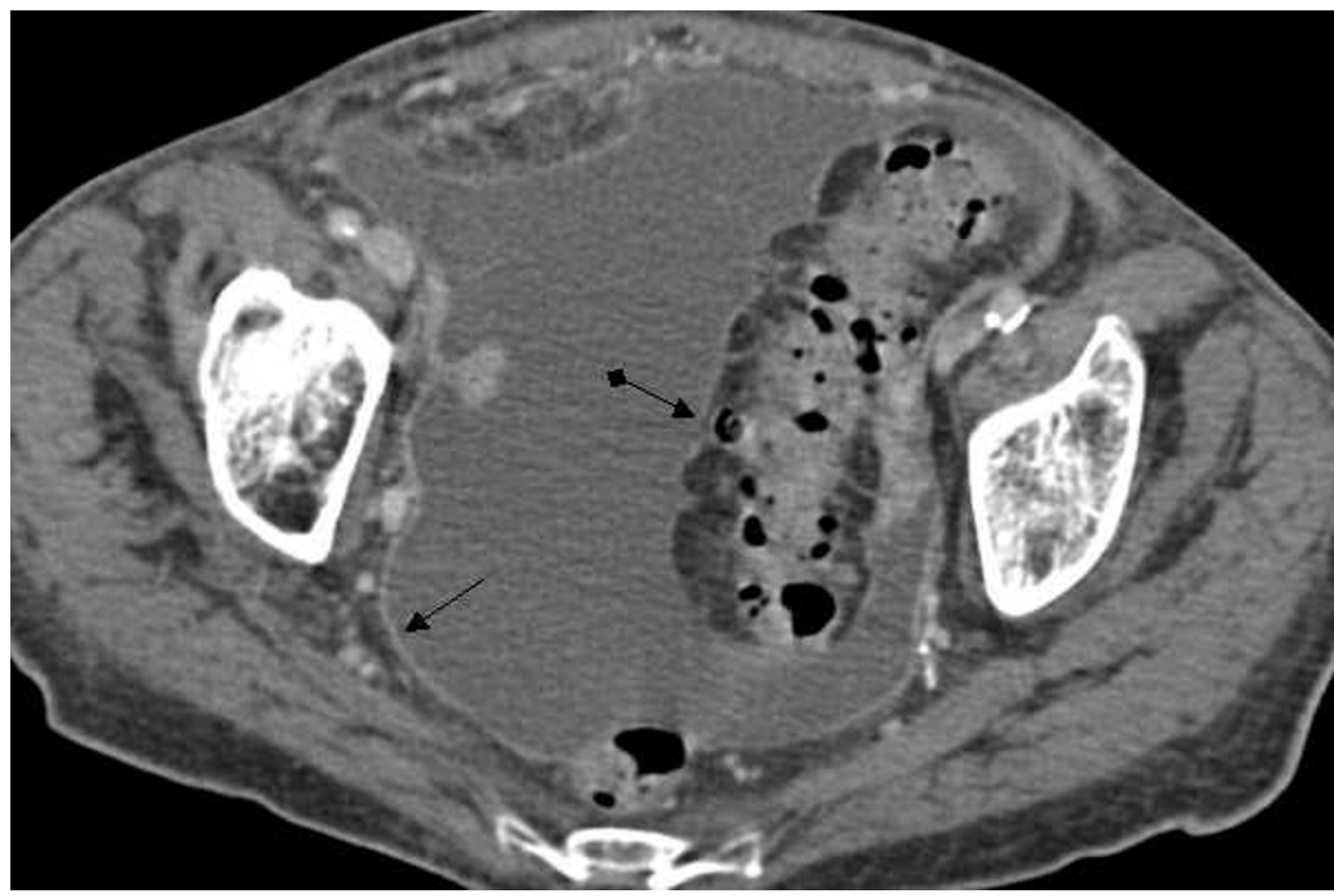

Figure I

CT pelvis pronounced contrast enhancement of the peritoneum

$\rightarrow$

; thickened wall of the sigmoid colon

infection and attacks the tubes during the sexually active period of life. It may be due to either a local extension from a tuberculous lymph node, Fallopian tube, tuberculous intestinal ulcer, or may be caused by hematogenous or lymphatic spread from distant sources of infection [4]. Although this patient had no previous medical history of pulmonary or extra-pulmonary tuberculosis, and the CT scan of the chest and abdomen showed no lymph node swellings anywhere, we are certain that the tuberculous infection was based on reactivation of a long-latent tuberculous focus in the peritoneum due to her immunocompromised state following a prolonged course of primary biliary cirrhosis over 14 years.

On the basis of the history, examination and laboratory findings a differential diagnosis of spontaneous bacterial peritonitis, bacterial cholangitis, intra-abdominal malignancy or abdominal tuberculosis was considered. The

\section{Table 2: Laboratory data of ascites on admission}

\begin{tabular}{ll}
\hline Protein & $4.6 \mathrm{mg} / \mathrm{dl}$ \\
Glucose & $60 \mathrm{mg} / \mathrm{dl}$ \\
LDH & $442 \mathrm{U} / \mathrm{l}$ \\
Cholesterol & $43 \mathrm{mg} / \mathrm{dl}$ \\
Leukocytes & $0.3 / \mathrm{nl}$ \\
\hline
\end{tabular}

patient was initially treated with high-dose broad-spectrum antibiotics. The patient's condition nevertheless continued to deteriorate and in addition to the CT and ultrasonographic findings, we had planned to perform a laparoscopy. During preparation for laparoscopy the culture of ascites returned positive for M. tuberculosis after three weeks.

Extrapulmonary manifestation of tuberculosis can be found in about $20.4 \%$ of cases in German population [5]. The incidence of tuberculous peritonitis in Germany has been very low and tuberculosis of the intestinal tract was found in approximately $0.8 \%$ of tuberculosis cases in 2004 [5]. The 'golden rule' for a rapid diagnosis of tuberculous peritonitis is a laparoscopy-guided biopsy. But because of the anaesthetic and bleeding risk, laparoscopyguided biopsy was not an immediately available option for our patient.

Positive cultures for $M$. tuberculosis have been reported from $7.8 \%$ in a small case report [6], up to $83 \%$ [7], which may be dependent on the fluid quantity. $1 \mathrm{~L}$ of fluid was recommended by Singh et al. [7].

The use of PCR to detect $M$. tuberculosis was diagnostically useful in patients with ascites who were suspected of hav- 
ing tuberculous peritonitis in order to achieve a prompter diagnosis and treatment. The IS6110 primer was detected in $60 \%$ of specimens $[8,9]$. Unfortunately the PCR analysis of ascitic fluid was not performed in this case.

Adenosine deaminase (ADA) levels are used for diagnosing tuberculosis in several locations and have also been recommended in suspected tuberculous peritonitis. The pertinent literature judges the usefulness of ADA levels in ascitic fluid as a diagnostic test for peritoneal tuberculosis differently. Riquelme et al. reported that ADA levels showed a high sensitivity (100\%) and specificity (97\%) in ascitic fluid using Giusti's methods [1]. Marinez-Vazquez reported that ADA is not specific for tuberculous peritonitis [10]. Lower sensitivities were reported in the context of underlying liver cirrhosis, and false positives occurred in malignancy and bacterial peritonitis [10,11]. ADA levels were not measured in this instance.

The question, why the tuberculin test was negative in this case, cannot be answered easily. New types of immunological test methods such as the Quanti FERON - TB Gold in - tube (ELISA assay) and the T - SPOT - TB test (ELISPOT assay), which are based on the interferon $\gamma($ IFN $-\gamma$ ) production of sensitized $\mathrm{T}$ lymphocytes, may yet provide a useful additional diagnostic method. In patients with extrapulmonary tuberculosis, a sensitivity of the IFN $-\gamma$ test of $92 \%$ was observed, although only 13 patients were included in the study [12]. Unfortunately, these methods were not available in Germany at the time the patient was admitted.

In this case, massive ascites was observed with multiple fine delicate septa on ultrasonography, and a thickened intestinal wall located in the sigmoid colon and pronounced enhancement of peritoneum was seen on CT scan. Case reports [13] and small case studies in the literature have already reported these findings retrospectively and prospectively [14-17].

Although tuberculous peritonitis may be associated with alcoholic cirrhosis of the liver, patients with PBC usually have ascites, making the diagnosis more difficult. At the time of diagnosis the decision to initiate anti-tuberculous therapy turned out to be difficult due to concomitant serious liver failure and no histological or bacteriological confirmation of infection with M. Tuberculosis. Five days after the therapy commenced the patient died of liver and multiple organ failure. In hindsight, an anti-tuberculous treatment should have been started without waiting for the culture report.

\section{Conclusion}

Tuberculous peritonitis must be considered in the initial differential diagnosis of patients with non-specific clinical signs and symptoms such as vomiting, abdominal pain, ascites, weight loss and fever that mimic the picture of spontaneous bacterial peritonitis in patients with PBC. The sonographic findings are not specific in tuberculous peritonitis, but can be useful in differentiating tuberculous ascites. An awareness of the ultrasonographic features may contribute valuable information, help in the diagnosis of tuberculous peritonitis, improve diagnostic accuracy and avoid clinical mismanagement.

\section{Abbreviations}

$\mathrm{ADA}=$ adenosine deaminase activity; $\mathrm{CT}=$ computed tomography; IFN $-\gamma=$ Interferon $\gamma ; \mathrm{M}=$ Mycobacterium; $\mathrm{PBC}=$ primary biliary cirrhosis; polymerase chain reaction $=$ PCR.

\section{Competing interests}

The author(s) declare that they have no competing interests.

\section{Authors' contributions}

YV was responsible for the patient's management; and manuscript design and drafting.

JB assisted with the manuscript draft and figures and provided general technical support.

GW was responsible for the radiological findings and provided the figures.

$\mathrm{BH}$ was responsible for the design, coordination and supervision of the patient's management.

All authors read and approved the final manuscript.

\section{Consent}

Written informed consent was obtained from the patient's relatives for the publication of the study.

\section{References}

I. Riquelme A, Calvo M, Salech F, Valderrama S, Pattillo A, Arellano M, Arrese M, Soza A, Viviani P, Letelier LM: Value of adenosine deaminase (ADA) in ascitic fluid for the diagnosis of tuberculous peritonitis: a meta-analysis. J Clin Gastroenterol 2006, 40(8):705-10.

2. Bernhard JS, Bhatia G, Knauer CM: Gastrointestinal tuberculosis: an eighteen-patient experience and review. J Clin Gastroenterol 2000, 30(4):397-402.

3. Khan R, Abid S, Jafri W, Abbas Z, Hameed K, Ahmad Z: Diagnostic dilemma of abdominal tuberculosis in non-HIV patients: an ongoing challenge for physicians. World J Gastroenterol I 2(39):637I-5. 2006, Oct 2I;

4. Sochocky S: Tuberculous peritonitis. A review of 100 cases. Am Rev Respir Dis 1967, 95(3):398-40I.

5. Brodhun B, Altmann D, Haas W: Report of epidemiology of tbc in Germany in 2004.

6. Demir K, Okten A, Kaymakoglu S, Dincer D, Besisik F, Cevikbas U, Ozdil S, Bostas G, Mungan Z, Cakaloglu Y: Tuberculous peritonitis - reports of 26 cases, detailing diagnostic and therapeutic problems. Eur J Gastroenterol Hepatol 200I, I3(5):58I-5. 
7. Singh MM, Bhargava AN, Jain KP: Tuberculous peritonitis. An evaluation of pathogenetic mechanisms, diagnostic procedures and therapeutic measures. $N$ Engl J Med 28 I (20): $109 \mid-4$. 1969, Nov 13:

8. Tzoanopoulos D, Mimidis K, Giaglis S, Ritis K, Kartalis G: The usefulness of PCR amplification of the IS6 I I 0 insertion element of M. tuberculosis complex in ascitic fluid of patients with peritoneal tuberculosis. Eur J Intern Med 2003, I4(6):367-37I.

9. Uzunkoy A, Harma M, Harma M: Diagnosis of abdominal tuberculosis: Experience from II cases and review of the literature. World J Gastroenterol I0(24):3647-3649. 2004 December I5;

10. Martinez-Vazquez JM, Ocana I, Ribera E, Segura RM, Pascual C: Adenosine deaminase activity in the diagnosis of tuberculous peritonitis. Gut 1986, 27(9): 1049-53.

1I. Hillebrand DJ, Runyon BA, Yasmineh WG, Rynders GP: Ascitic fluid adenosine deaminase insensitivity in detecting tuberculous peritonitis in the United States. Hepatology 1996, 24(6): | 408-I 2.

12. Ravn P, Munk ME, Andersen AB, Lundgren B, Lundgren JD, Nielsen LN, Kok-Jensen A, Andersen P, Weldingh K: Prospective evaluation of a whole-blood test using Mycobacterium tuberculosis-specific antigens ESAT- 6 and CFP-10 for diagnosis of active tuberculosis. Clin Diagn Lab Immonol 2005, I 2(4):49I-6.

13. Makiyama A, Okuyama Y, Okajima T, Fujimoto S: Tuberculous peritonitis. J Gastroenterol 2003, 38( I 2): I | 67-70.

14. Yilmaz T, Sever A, Gur S, Killi RM, Elmas N: CT findings of abdominal tuberculosis in 12 patients. Comput Med Imaging Graph 2002, 26(5):32I-5.

15. Rodriguez E: Pom. Peritoneal tuberculosis versus peritoneal carcinomatosis: distinction based on CT findings. J Comput Assist Tomogr 1996, 20(2):269-72.

16. Lee DH, Lim JH, Ko YT, Yoon Y: Sonographic findings in Tuberculous peritonitis of wet-ascitic type. Clinical Radiology 1991, 44:306-310.

17. Akhan O, Demirkazik FB, Demirkazik F, Gulekon N, Eryilmaz M, Unsal M, Besim A: Tuberculous peritonitis: ultrasonic diagnosis. J Clin Ultrasound 1990, 18:7| I-7|4.

Publish with Bio Med Central and every scientist can read your work free of charge

"BioMed Central will be the most significant development for disseminating the results of biomedical research in our lifetime. "

Sir Paul Nurse, Cancer Research UK

Your research papers will be:

- available free of charge to the entire biomedical community

- peer reviewed and published immediately upon acceptance

- cited in PubMed and archived on PubMed Central

- yours - you keep the copyright

Submit your manuscript here:

http://www.biomedcentral.com/info/publishing_adv.asp
Biomedcentral 